\title{
Rescue and evaluation of a recombinant PRRSV expressing porcine Interleukin-4
}

\author{
Zhijun Li ${ }^{1,2}$, Gang Wang ${ }^{3}$, Yan Wang ${ }^{1,2}$, Chong Zhang ${ }^{3}$, Xinglong Wang ${ }^{1,2}$, Baicheng Huang ${ }^{1,2}$, Qiongyi Li ${ }^{1,2}$, \\ Liangliang $\mathrm{Li}^{1,2}$, Biyun Xue ${ }^{1,2}$, Peiyang Ding ${ }^{1,2}$, Shahid Faraz Syed ${ }^{1,2}$, Chengbao Wang ${ }^{1,2^{*}}$, Xuehui Cai ${ }^{3^{*}}$ \\ and En-Min Zhou ${ }^{1,2^{*}}$
}

\begin{abstract}
Background: The current vaccines for porcine reproductive and respiratory syndrome virus (PRRSV) have failed to provide broad protection against infection by various strains of PRRSV. Porcine Interleukin-4 (plL-4) plays an important role in the regulation of the immune response and has been used previously as an immunological adjuvant. The objective of this study was to construct a recombinant PRRSV expressing pIL-4 and to evaluate the immune response of the recombinant virus in piglets.

Methods: The plL-4 gene was inserted in the PRRSV (CH-1R strain) infectious clone by overlap PCR. Indirect immunofluorescence assay (IFA) and Western blotting were used to confirm the recombinant virus. The stability of the recombinant virus was assessed by DNA sequencing and IFA after 15 passages in vitro. Recombinant virus was injected into pigs and efficacy of immune protection was evaluated in comparison with the parental virus.

Results: The recombinant virus ( $\mathrm{CH}-1 \mathrm{R} / \mathrm{plL}-4)$ was successfully rescued and shown to have similar growth kinetics as the parental virus. The recombinant virus was stable for 15 passages in cell culture. Pigs vaccinated with CH-1R/plL-4 produced a similar humoral response to the response elicited by parental virus, but IL-4 level in the supernatant of PBMCs from pigs vaccinated with $\mathrm{CH}-1 \mathrm{R} / \mathrm{plL}-4$ was significantly higher than the parent virus at 28 days post-immunization (DPI). Flow cytometric (FCM) analysis showed that the percentage of $\mathrm{CD}^{+} \mathrm{CD}^{+}$double positive T (DPT) cells in the $\mathrm{CH}-1 \mathrm{R} / \mathrm{plL}-4$ vaccinated group was significantly higher than the parental virus at 3 and 7 Days Post-Challenge (DPC), and the IL-4 level in the blood significantly increased at 7 DPC. However, the viral load and histopathology did not show significant difference between the two groups.

Conclusions: A recombinant PRRSV expressing porcine IL-4 was rescued and it remained genetically stable in vitro. The recombinant virus induced higher DPT ratios and IL-4 levels in the blood after HP-PRRSV challenge compared to the parental virus in piglets. However, it did not significantly improve protection efficacy of PRRSV vaccine.
\end{abstract}

Keywords: PRRSV, Reverse genetic technology, Porcine IL-4, Vaccine

\section{Background}

Porcine reproductive and respiratory syndrome (PRRS) is characterized by clinical signs of reproductive failure in pregnant sows and respiratory distress in pigs of all ages [1]. Since it's first reported in 1987, PRRS has become a severe threat to the swine industry worldwide [2]. The

\footnotetext{
*Correspondence: wangchengbao@nwsuaf.edu.cn; aci139@sina.com; zhouem@nwsuaf.edu.cn

'Department of Preventive Veterinary Medicine, College of Veterinary Medicine, Northwest A\&F University, Yangling, Shaanxi 712100, China

${ }^{3}$ State Key Laboratory of Veterinary Biotechnology, Harbin Veterinary Research Institute of Chinese Academy of Agriculture Science, Harbin 150001, China

Full list of author information is available at the end of the article
}

causative agent of PRRS, the porcine reproductive and respiratory syndrome virus (PRRSV) was successfully isolated in EU and USA in 1991 and 1992, respectively $[3,4]$. Surprisingly, the two genotypes showed sequence identity of only $60 \%$ and a high degree of antigenic variation [5, 6]. In June 2006, an unparalleled, large-scale, PRRS outbreak occurred in China, leading to widespread infection which continues to be a serious problem today. Several studies have confirmed that a unique highly pathogenic strain of PRRSV (HP-PRRSV) was responsible for this outbreak [7-9]. 
PRRSV is an enveloped virus with a single-stranded, positive-sense RNA genome of approximately $15 \mathrm{~kb}$ in length [10]. The genome contains a cap structure at the 5' end, a poly (A) tail at the $3^{\prime}$ end, and $5^{\prime}$ and $3^{\prime}$ un-translated regions (UTRs). Two overlapping open reading frames (ORF 1a and ORF 1b) occupy two thirds of the genome at the $5^{\prime}$ end. These ORFs encode two large polyproteins that undergoes co-translational and posttranslational processing to give rise to at least 14 nonstructural proteins (nsps). ORFs 2-7 code for viral structural proteins GP2, E, GP3, GP4, GP5a, GP5, M, and $\mathrm{N}$, respectively [11-14]. According to the recently accepted replication model for viruses of the order Nidovirales, PRRSV and other arteriviruses synthesize a nested set of subgenomic (sg) mRNAs as a mechanism to regulate the expression of structural or accessory proteins [15]. Base pair interactions between the transcription-regulating sequences (TRSs) located at the 3' end of the leader sequence and the complement of body TRSs presented upstream of each gene, have been shown to be essential for this process [16].

It is well recognized that due to the genetic diversity of the PRRSV strains, none of the current vaccines can completely protect against PRRSV infections. For PRRSV vaccines, the efficacy of protective immunity is usually assessed by the reduction in viremia after challenge with a virulent virus $[17,18]$. Sufficient amount of neutralizing antibodies may prevent the infection, but cannot clear the virus in blood during the course of the infection. Therefore, vaccine immunity relies on the induction of the protective cellmedicated immune responses in prevention against PRRSV infection $[19,20]$. $\mathrm{CD}^{+} \mathrm{CD}^{+}$double positive T (DPT) cells are the main effectors of adaptive cellular immune response and essential to clear viral infection [21]. IFN- $\gamma$ secreting cells are mainly DPT cells and whose levels are correlated with the host ability prevent PRRSV infection [22].

It is clear that there is an urgent need for a safe and more effective PRRSV vaccine. In order to increase the efficacy of the vaccine, an alternative approach is to co-deliver cytokines to up-regulate the immune response of vaccine antigens. Interleukin-4 (IL-4) is a pleiotropic cytokine and has been used as an adjuvant to enhance immune response of PRRSV vaccines [23, 24]. In addition, IL-4 may positively modulate vaccination mediated clearance of PRRSV [22, 25]. In this study, porcine Interleukin-4 (pIL-4) gene was inserted between the $\mathrm{N}$ gene and the 3 '-UTR of a live attenuated PRRSV infectious clone, along with a copy of the transcription regulating sequence for ORF6 (TRS6), to create a recombinant PRRSV. The growth characterization and genetic stability of the recombinant virus was first analyzed in vitro. Further, recombinant virus was injected into piglets to evaluate its potential role for improving vaccine efficacy in protection against HP-PRRSV infection.

\section{Results}

\section{Rescue of recombinant virus harboring plL-4}

The recombinant plasmid pBAC-CH-1R/pIL-4 (Fig. 1) was constructed according to the primers as shown in Table 1 . The recombinant virus $\mathrm{CH}-1 \mathrm{R} / \mathrm{pIL}-4$ was successfully rescued after transfecting pBAC-CH-1R/pIL-4 plasmid into Marc-145 cells. CPE were markedly observed after 3 days post-transfection (data not shown), supernatants were then harvested and passaged 3 times in Marc-145 cells.

The presence of recombinant PRRSV was identified by indirect immunofluorescence assay (IFA) and western blotting using the PRRSV N-specific antibody (6D10). Expression of pIL-4 was confirmed using a goat anti-pIL-4 polyclonal Ab. As shown in Fig. 2a, the presence of the virus was confirmed by the visualization of red fluorescence, and pIL-4 with green fluorescence. Western blotting was used to verify protein expression of $\mathrm{N}$ and IL-4 (Fig. 2b).

As expected, pIL-4 expression via an additional TRS did not influence the growth characteristics of the virus. As shown in Fig. 2c, growth rate and maximum titer of the

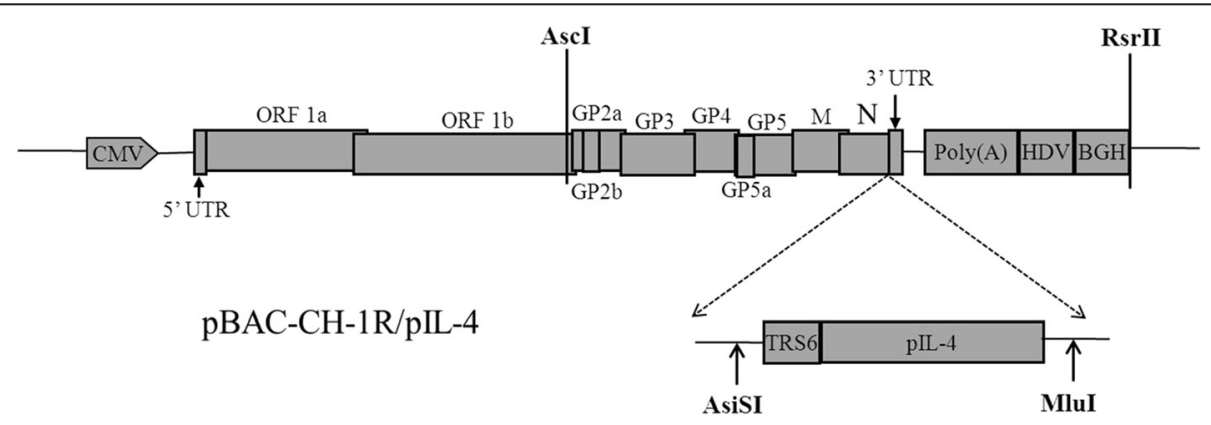

Fig. 1 Schematic diagram of the recombinant plasmid (pBAC-CH-1R/plL-4). The porcine IL-4 (plL-4) gene was inserted between the $\mathrm{N}$ gene and the 3'-UTR of the attenuated PRRSV infectious clone (pBAC-CH-1R). It exhibited expression as a separate sub-genomic RNA with a copy of the transcription regulation sequence for ORF6 (TRS6) 
Table 1 Primers used for construction and identification of recombinant PRRSV infectious clones

\begin{tabular}{lll}
\hline Primers & Nucleotide sequence (5'-3') & Sense \\
\hline F1-1 & TAACCAGAGTTCAGCGGACAATGGGTCTCACCTCCCAAC \\
F1-2 & GCGATCGCTGATGGTTCCGTGGCAACCCCTTAACCAGAGTTCATG \\
R1 & CGACGCGTCGTCAACACTTGAGTATTTCTCCTT \\
F2 & ATACTGTGCGCCTGATCCGC \\
R2 & TCGCCAATTAAACTTACCCCCACA & + \\
\hline
\end{tabular}

The italics in the sequence denote the restriction enzyme sites (Asis I, Mlu I)

recombinant virus were similar to those of the parental virus. Titers peaked at $48 \mathrm{~h}$ post infection (hpi) for both viruses.

The expression of pIL-4 was further analyzed by determining the accumulation kinetics of pIL- 4 in the supernatant of infected Marc-145 cells. The concentration of IL-4 was $300 \mathrm{pg} / \mathrm{mL}$ at $24 \mathrm{hpi}$, and it reached the maximum concentration of $800 \mathrm{pg} / \mathrm{mL}$ at $48 \mathrm{hpi}$ (Fig. 2d).

\section{Genetic stability testing}

The recombinant virus was serially passaged in Marc145 cells for 15 times to examine the genetic stability of pIL-4 in the virus over time. Viral RNA was isolated from cells after passage 5, 10 and 15 post-infection with recombinant virus. The presence of the pIL-4 gene in the viral genome was confirmed using RT-PCR. Unexpected gene insertion, deletion and mutation were ruled out by sequencing (data not shown).

\section{Humoral immune responses}

The host humoral immune response was assessed in pigs at various time-points (Fig. 3a). The antibody titers of the pigs in the two vaccinated groups showed similar trend and they could be initially detected at 21 DPI. Titers significantly increased at 7 through $21 \mathrm{DPC}$ and then dropped at 28 DPC. Antibodies for the challenged (non-vaccinated)
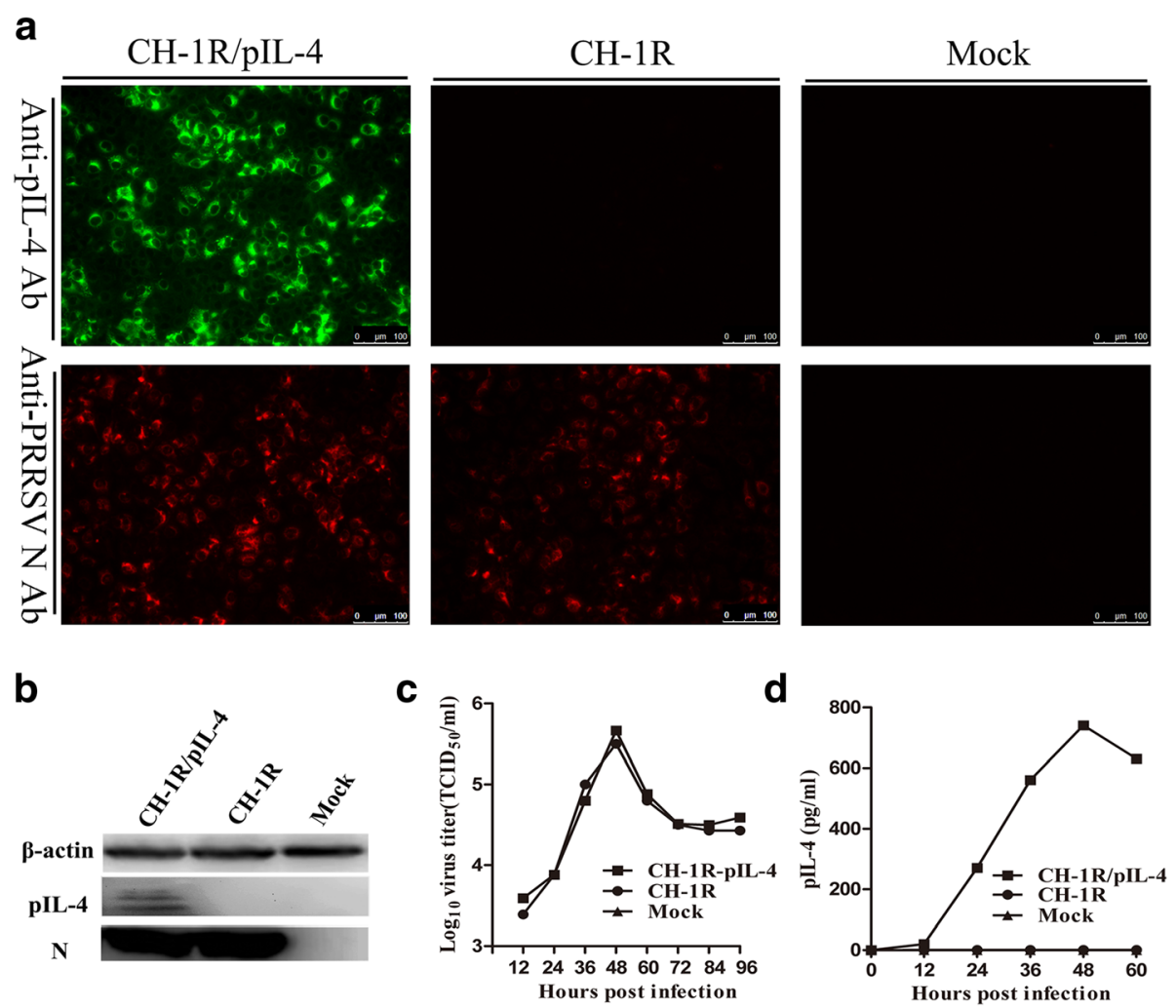

Fig. 2 Characterization of recombinant PRRSV expression of the plL-4 gene in Marc-145 cells. a IFA detection of plL-4 and N expression in Marc-145 cells infected with parental virus or recombinant virus. Original magnification 200x. b Western blot detection of plL-4 and N protein in cell lysates of Marc-145 cells infected with recombinant virus and parental virus. c Growth curves of the recombinant and the parental virus in Marc-145 cells. $\mathbf{d}$ Kinetics of plL-4 accumulation in the supernatants of Marc-145 cells infected with the recombinant virus vs. parental virus or mock control. Data are represented as the mean of three independent experiments 

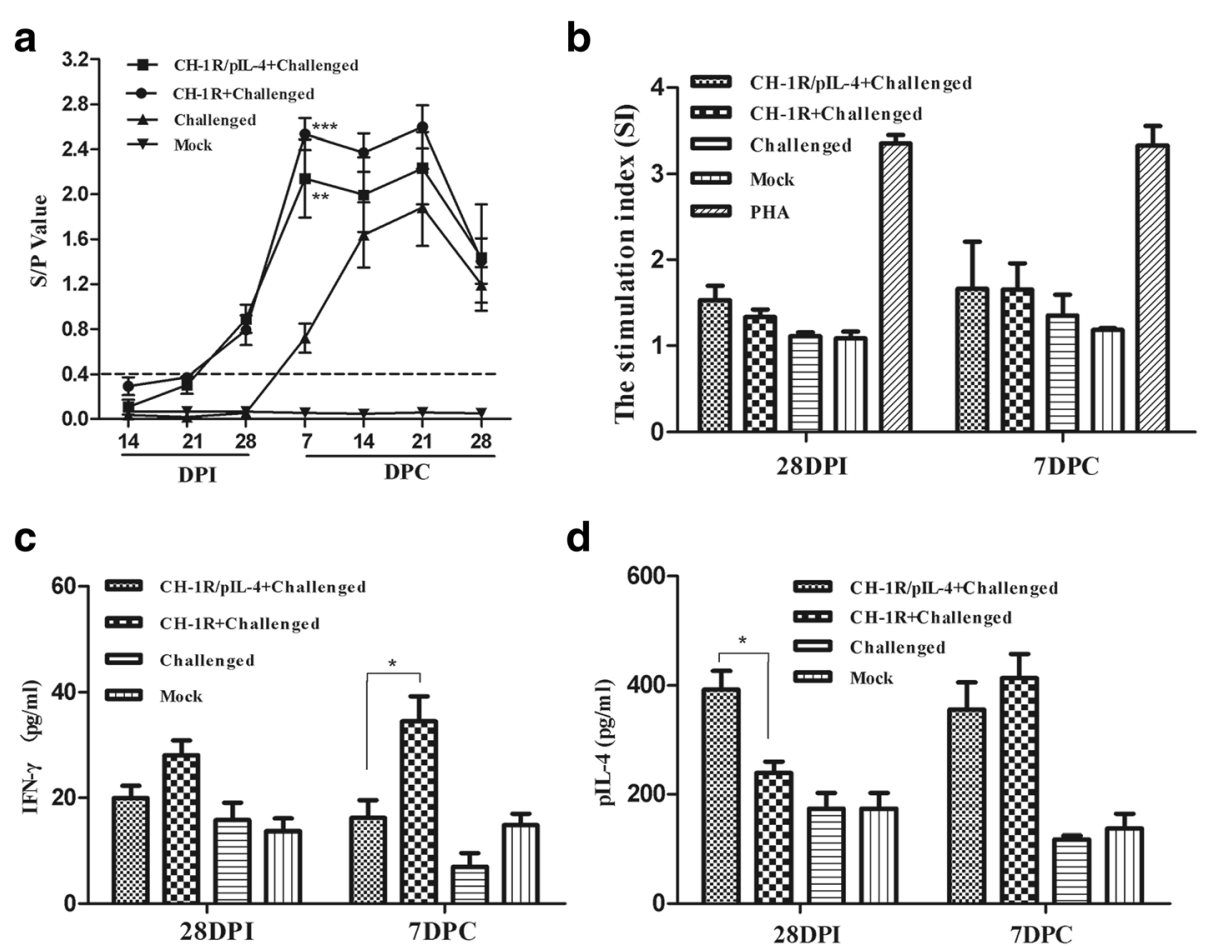

Fig. 3 Humoral and cell-mediated immunity in pigs infected with the recombinant and the parental virus. a PRRSV-specific serum antibodies were measured with the IDEXX HerdChek ${ }^{\oplus}$ PRRSV ELISA 2XR Kit. S/P ratios of greater than 0.4 are considered positive. S/P = (Sample A (650)-Negative Control) / (Positive Control- Negative Control). Parental and recombinant virus groups were compared to the challenged control group. ${ }^{* *}$ denotes $p<0.01$; *** represents $p<0.001$. b PBMCs were collected from the piglets stimulated with purified PRRS virions. PHA was the positive control. After $72 \mathrm{~h}$ of stimulation, the cells were treated with WST-8 for $2 \mathrm{~h}$ and the $\mathrm{OD}_{450}$ values were determined to calculate the stimulation index (SI). IFN- $\gamma(\mathbf{c})$ and IL-4 (d) concentrations in the supernatants of PBMC cultures were tested after $72 \mathrm{~h}$ of stimulation using commercial ELISA Kits. *indicates $p<0.05$. Data represent the mean \pm SD of five pigs from each group

control group were initially detected at 7 DPC. The titers were markedly increased by $21 \mathrm{DPC}$ and then dropped at 28 DPC. Virus titers were significantly higher at 7 DPC in challenged unvaccinated group than in the groups vaccinated with parental or recombinant virus $(p<0.01$, $p<0.001$, respectively). No seroconversion was evident in the mock group.

\section{T lymphocyte proliferation assay}

Purified PRRSV $\mathrm{CH}-1 \mathrm{R}$ antigen was used as stimulator to perform lymphocyte proliferation assay. As shown in Fig. $3 \mathrm{~b}$, the $\mathrm{CH}-1 \mathrm{R} / \mathrm{pIL}-4$ vaccinated group stimulated PRRSV-specific lymphocyte proliferative response which was similar to the response of the $\mathrm{CH}-1 \mathrm{R}$ vaccinated group at 28 DPI and 7 DPC.

We further monitored the temporal expression of INF- $\gamma$ and IL-4 in PBMCs after stimulation with purified PRRSV. As shown in Fig. 3c, IFN- $\gamma$ expression in PBMCs from pigs in the $\mathrm{CH}-1 \mathrm{R} / \mathrm{pIL}-4$ group was significantly lower than expression in the $\mathrm{CH}-1 \mathrm{R}$ vaccinated group at 7 DPC $(p<0.05)$. However, IL-4 expression was significantly higher in the $\mathrm{CH}-1 \mathrm{R} / \mathrm{pIL}-4$ group than in the $\mathrm{CH}-1 \mathrm{R}$ group at $28 \mathrm{DPI}(p<0.05)$ (Fig. $3 \mathrm{~d})$.

\section{T lymphocyte phenotypic analysis}

The percentages of $\mathrm{CD}^{+}$single positive $\mathrm{T}$ (SPT) cells, $\mathrm{CD}_{4}^{+}$SPT cells, $\mathrm{CD}^{+}$SPT cells, and $\mathrm{CD}^{+} \mathrm{CD}^{+}$double positive $\mathrm{T}$ (DPT) cells in peripheral blood were measured by flow cytometric (FCM) analysis. The percentage of CD3 + SPT cell subpopulations remained relatively stable in all groups during the experimental period. There was no significant change in the levels of the DPT cells in either of the immunized groups during vaccination period, however, the numbers of DPT cells in the recombinant virus group were markedly higher at 3 and 7 DPC compared with the parental virus group $(p<0.05)$. Interestingly, the DPT cells in the challenged control group rapidly decreased after challenge, returned to normal by 21 DPC. Table 2 shows the complete results of the FCM analysis.

\section{Cytokine responses}

At 14 DPI and 0, 7, 14, 21 and 28 DPC, the sera of the pigs were collected to detect the production of IFN- $\gamma$ and IL-4. As shown in Fig. 4a, IFN- $\gamma$ expression in sera was not significantly different between two immunized groups during experimental period. However, mean IL-4 level in the serum of group vaccinated with recombinant 
Table 2 Flow cytometric analysis for proportion of T lymphocyte subpopulations in peripheral blood of pigs

\begin{tabular}{|c|c|c|c|c|c|c|c|}
\hline Marker & $14^{\mathrm{a}}$ & $28^{\mathrm{a}}$ & $3^{b}$ & $7^{b}$ & $14^{\mathrm{b}}$ & $21^{b}$ & $28^{b}$ \\
\hline \multicolumn{8}{|l|}{$\mathrm{CD}^{+}$} \\
\hline Group1 & $56.4 \pm 4.5$ & $61.1 \pm 8.6$ & $65.6 \pm 3.6$ & $66.4 \pm 5.0$ & $57.7 \pm 4.7$ & $58.7 \pm 7.0$ & $69.8 \pm 7.9$ \\
\hline Group2 & $51.9 \pm 5.6$ & $49.0 \pm 6.7$ & $53.9 \pm 6.8$ & $59.9 \pm 3.9$ & $51.8 \pm 8.4$ & $54.4 \pm 5.8$ & $58 \pm 6.6$ \\
\hline Group3 & $52.7 \pm 3.9$ & $49.8 \pm 10.9$ & $44.76 \pm 6.3$ & $59.6 \pm 8.8$ & $61.1 \pm 5.2$ & $57.2 \pm 7.3$ & $51.4 \pm 8.3$ \\
\hline Group4 & $50.8 \pm 2.1$ & $49.8 \pm 6.4$ & $48.3 \pm 7.0$ & $52.6 \pm 8.7$ & $51.8 \pm 10.7$ & $50.6 \pm 9.7$ & $52.4 \pm 6.8$ \\
\hline \multicolumn{8}{|l|}{$\mathrm{CD}^{+}$} \\
\hline Group1 & $18.6 \pm 3.4$ & $15 \pm 4.1$ & $9.7 \pm 3.8$ & $4.6 \pm 0.3$ & $4.3 \pm 4.2$ & $1.9 \pm 0.6$ & $4.1 \pm 1.2$ \\
\hline Group2 & $17 \pm 1.2$ & $16.5 \pm 8.4$ & $5.9 \pm 2.9$ & $5.2 \pm 5.4$ & $6.3 \pm 2.3$ & $1.33 \pm 0.9$ & $4.2 \pm 2.5$ \\
\hline Group3 & $28.7 \pm 0.2$ & $24.9 \pm 8.2$ & $11.6 \pm 5.5$ & $3.1 \pm 1.5$ & $8.0 \pm 5.3$ & $20 \pm 6.3$ & $22.7 \pm 2.2$ \\
\hline Group4 & $23.6 \pm 4.3$ & $30.7 \pm 5.4$ & $26.5 \pm 3.7$ & $32.4 \pm 0.2$ & $26.3 \pm 3.5$ & $19.6 \pm 2.8$ & $23.1 \pm 3.3$ \\
\hline \multicolumn{8}{|l|}{$\mathrm{CD8}^{+}$} \\
\hline Group1 & $30.3 \pm 5.3$ & $33.3 \pm 8.1$ & $27.6 \pm 6.5$ & $64.8 \pm 8.2$ & $50.7 \pm 6.6$ & $53.0 \pm 9.2$ & $46 \pm 0.2$ \\
\hline Group2 & $27.5 \pm 5.4$ & $35.5 \pm 7.3$ & $26.4 \pm 3.0$ & $58.8 \pm 9.1$ & $48.6 \pm 2.0$ & $51.8 \pm 11.2$ & $50.1 \pm 4.7$ \\
\hline Group3 & $28.0 \pm 4.5$ & $29.1 \pm 7.5$ & $13.5 \pm 2.1$ & $24.5 \pm 6.2$ & $29.7 \pm 9.5$ & $38.8 \pm 3.2$ & $30 \pm 3.6$ \\
\hline Group4 & $29.7 \pm 6.4$ & $32.7 \pm 3.0$ & $29.3 \pm 2.6$ & $29.5 \pm 8.7$ & $30.2 \pm 8.9$ & $32.4 \pm 3.3$ & $36.7 \pm 0.5$ \\
\hline \multicolumn{8}{|c|}{$\mathrm{CD}^{+} \mathrm{CD}^{+}$} \\
\hline Group1 & $29.4 \pm 7.8$ & $23.9 \pm 8.6$ & $32.9 \pm 3.1^{*}$ & $39.1 \pm 2.6^{*}$ & $28.3 \pm 7.1$ & $25.4 \pm 6.5$ & $31.4 \pm 7.7$ \\
\hline Group2 & $30.5 \pm 4.2$ & $21.7 \pm 9.7$ & $18.3 \pm 5.2$ & $26.4 \pm 4.5$ & $17.2 \pm 7.4$ & $26.0 \pm 7.9$ & $29.5 \pm 3.8$ \\
\hline Group3 & $18.9 \pm 0.1$ & $18.5 \pm 7.0$ & $8.7 \pm 3.0$ & $7 \pm 5.7$ & $13.2 \pm 1.2$ & $19.2 \pm 6.9$ & $18.1 \pm 5.3$ \\
\hline Group4 & $20.5 \pm 4.3$ & $22.8 \pm 3.1$ & $28 \pm 4.8$ & $26.4 \pm 10.2$ & $25.7 \pm 8.0$ & $23.5 \pm 6.5$ & $28.9 \pm 4.1$ \\
\hline
\end{tabular}

Results are expressed as mean (\%) \pm SD. Group 1: CH-1R/plL-4 + Challenged; Group 2: $\mathrm{CH}-1 \mathrm{R}+$ Challenged; Group 3: Challenged; Group 4: Mock. ${ }^{*} p<0.05$ ${ }^{\text {a}}$ Days post- immunization (DPI); ${ }^{\mathrm{b}}$ Days post-challenge (DPC)

virus was significantly higher than that of parental virus group at 7 DPC $(p<0.01)$ (Fig. $4 \mathrm{~b})$.

Observation of clinical signs and rectal temperature testing All of the pigs presented with a high fever $\left(\geq 40{ }^{\circ} \mathrm{C}\right)$ after challenge. Rectal temperatures from the challenged group increased rapidly at $2 \mathrm{DPC}$ and remained above $41{ }^{\circ} \mathrm{C}$ from 3 to $12 \mathrm{DPC}$, before returning to normal at $14 \mathrm{DPC}$. Two immunized groups also showed similar fever pattern, but the overall rectal temperatures were relatively low and of shorter duration. There was no significant difference in fever magnitude and duration between groups. No rectal temperature variations were observed in the mock group during the experimental period (Fig. 5a).

The challenged group showed obvious clinical signs of infection, including dyspnea, severe lethargy, cough, and anorexia after challenge. Two pigs showed typical "blue ears" and died at 7 and 10 DPC. Similar clinical signs were observed in the two immunized groups following challenge, but the signs were milder and of shorter duration relative to the unimmunized pigs. The scores of clinical signs between two immunized groups were
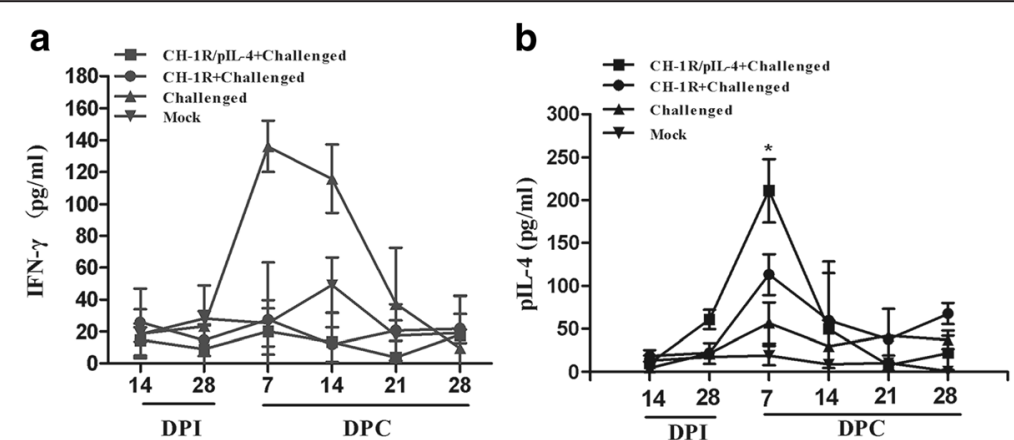

Fig. 4 Concentrations of Th1-type (IFN- $\gamma$ ) and Th2-type (IL-4) cytokines in the sera of pigs. Serum samples were collected from five pigs in each group at different time points and quantitative ELISA kits were used to detect level of IFN- $\gamma(\mathbf{a})$ or IL-4 (b). The CH-1R vaccinated group was compared to the recombinant vaccinated group and * denotes $p<0.05$. Data represent the mean \pm SD of five pigs from each group 


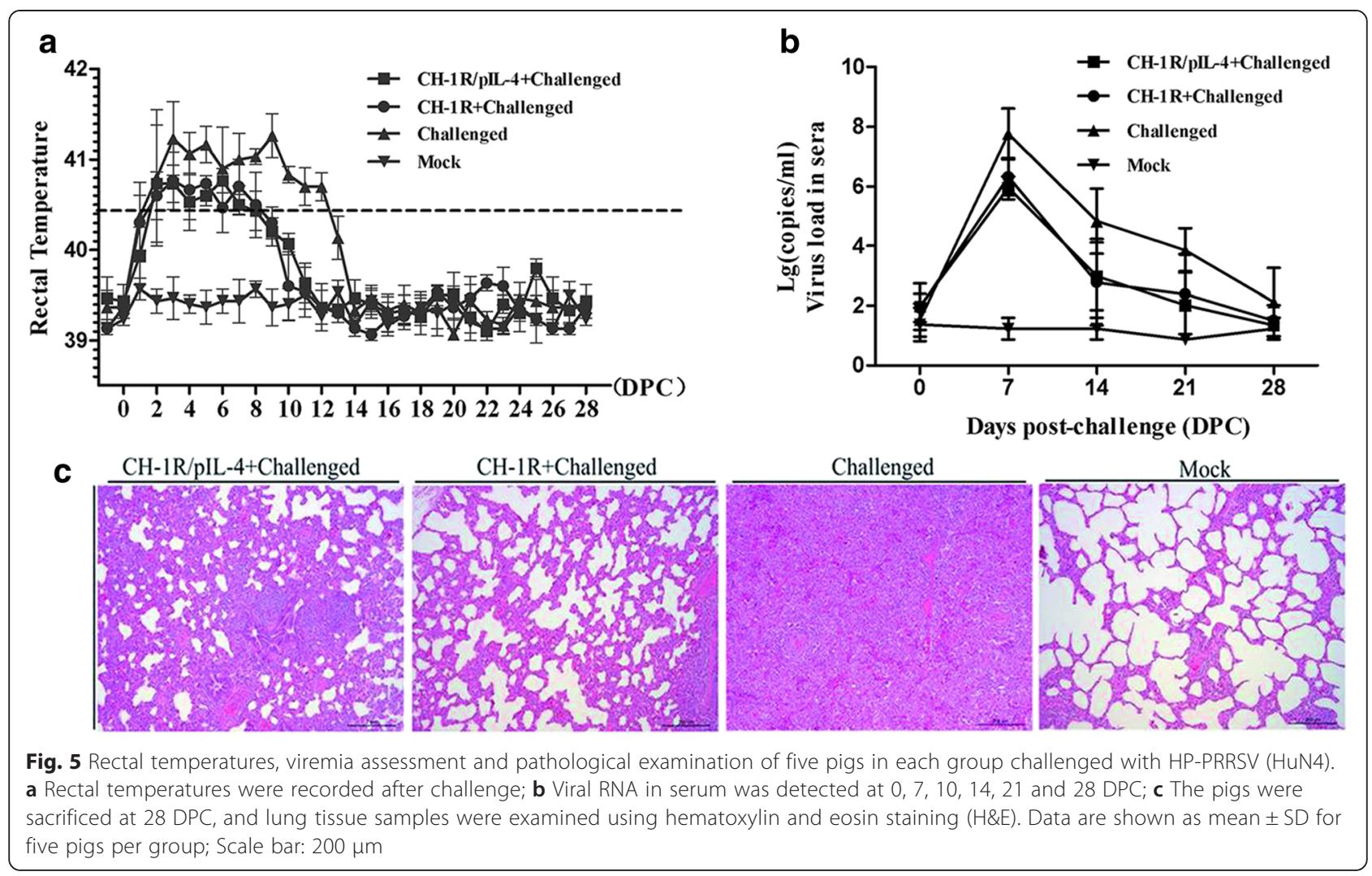

not significant difference $(p>0.05)$, but were significantly lower than that in the challenged control group $(p<0.05)$ (Table 3).

\section{Viremia assessment}

At $0,7,14,21$ and $28 \mathrm{DPC}$, the blood samples of the pigs were collected and the viral loads in the sera were investigated. As shown in Fig. 5b, all of the challenged groups developed highest degree of viremia at 7 DPC, gradually dropped at $14 \mathrm{DPC}$ and then returned to normal. The two immunized groups exhibited slightly less viremia than the un-immunized challenge group, but no significant difference was observed $(p>0.05)$.

Table 3 The scores of clinical signs after challenge and lung lesions recorded at $28 \mathrm{DPC}^{\mathrm{a}}$

\begin{tabular}{lcr}
\hline Groups & $\begin{array}{l}\text { Scores of clinical } \\
\text { signs }( \pm \mathrm{SD})^{\mathrm{b}}\end{array}$ & $\begin{array}{r}\text { Scores of lung } \\
\text { lesions }( \pm \mathrm{SD})^{\mathrm{C}}\end{array}$ \\
\hline $\mathrm{CH}-1 \mathrm{R} /$ plL-4 + Challenged & $3.9 \pm 0.18 \mathrm{~B}$ & $21.5 \pm 3.32 \mathrm{~B}$ \\
$\mathrm{CH}-1 \mathrm{R}+$ Challenged & $3.4 \pm 0.27 \mathrm{~B}$ & $23.6 \pm 5.57 \mathrm{~B}$ \\
Challenged & $5.6 \pm 1.58 \mathrm{~A}$ & $51.2 \pm 8.33 \mathrm{~A}$ \\
Mock & $3 \pm 0.00 \mathrm{~B}$ & $6.67 \pm 0.58 \mathrm{C}$ \\
\hline
\end{tabular}

${ }^{a}$ Within each column, the different letters $(A, B, C)$ represent the values significantly different $(p<0.05)$

${ }^{\mathrm{b}}$ Scores of clinical sign were expressed as the sum of daily observations of all clinical signs

${ }^{c}$ Percentage of the entire lung affected by pneumonia

\section{Pathological examination}

On histological examination, lung lesions of pigs in the challenged group exhibited alveolar epithelial cell proliferation and extensive infiltration of inflammatory cells. However, the interstitial pneumonitis observed in the $\mathrm{CH}-1 \mathrm{R} / \mathrm{pIL}-4$ or $\mathrm{CH}-1 \mathrm{R}$ vaccinated groups were milder than that of un-immunized challenged group at 28 DPC. The mock group was normal (Fig. 5c).

\section{Discussion}

The non-essential regions of the PRRSV nsp2 gene have been utilized previously as an insertion site for expressing a foreign gene [26-31], but stability of the recombinant virus was a concern [32]. As a potential remedy to this problem, foreign gene was inserted between PRRSV ORF1b and ORF2a, along with a copy of TRS. This approach had particular advantage of minimizing the effects of expression and post-translational modification of viral proteins [33], and a series of recombinant PRRS viruses have been constructed using this approach [33-37]. In this study, the pIL- 4 gene was inserted between $\mathrm{N}$ gene and 3'-UTR of a live attenuated PRRSV infectious clone using a similar strategy. Both recombinant virus and parental virus exhibited similar virus titers after growth in Marc145 cells, and this pattern was critical for recombinant vaccine development [38]. 
Previous studies demonstrated that pIL-4 markedly enhanced the protective immune response of pigs and improved the efficacy of the MLV in preventing PRRS disease [23], and pIL-4 can enhance immunogenicity of the PRRSV ORF5 gene vaccine [24]. Conversely, however, pigs that received PRRSV ORF7 DNA vaccine plus pIL-4encoded plasmids did not develop any improved humoral and cellular immune responses [39]. In present study, recombinant virus expressing pIL-4 did not significantly enhance the protective immune response after vaccination. Thus, observations on the adjuvant effect of pIL-4 on PRRSV vaccine were inconsistent, which may be related to the species. Porcine IL-4 cannot be used as immunological adjuvant since it was reported that IL-4 plays a different role in pigs than in mice and humans, and porcine IL-4 blocks antibody production and also suppresses antigenstimulated proliferation of B cells [40]. Another potential reason may be attributed to insufficient expression levels of IL-4 or inefficiency of IL-4 in potentiating anti-PRRSV immunity in vivo. In the absence of adequate amount, IL4 cannot work efficiently as adjuvant to enhance immunity efficacy or prevent PRRSV infection [41]. Thus, additional studies on IL-4 as an adjuvant are required in different types of PRRSV vaccine as well as in other veterinary vaccines to evaluate its adjuvant property.

In this study, we monitored the percentage of several $\mathrm{T}$ lymphocyte subpopulations in the blood at different time. Our data showed that the frequency of total $\mathrm{CD}^{+}$ $\mathrm{T}$ subpopulations of pigs from all groups was relatively stable during experimental period (Table 2). Conversely, a rapid down-regulation of $\mathrm{CD} 4^{+} \mathrm{CD} 8^{+}$double positive $\mathrm{T}$ (DPT) cells was observed in the challenged control group after HP-PRRSV (HuN4) infection, as previously reported $[42,43]$. These results suggest one mechanism of the PRRSV-mediated delay in initiation of virus-specific adaptive immunity is by altering frequency of important lymphocytes early post-infection. In addition, we also observed that DPT cells in the recombinant virus group were markedly higher than the parental virus group at 3 and 7 DPC. Meanwhile, mean IL-4 level in the serum of group vaccinated with recombinant virus was significantly higher than that from parental virus group at 7DPC (Fig. 4b). IL-4 is a mediator of DPT cells cross-talk leading towards the development of immunity against an infectious pathogen [44], and IL-4 had a potent positive influence on the generation of DPT cells originally cloned from $\mathrm{CD}^{+} \mathrm{CD}^{-} \mathrm{T}$ lymphocytes $[45,46]$. Our results also indicated that the higher level of IL-4 may be helpful to increase the DPT ratio in the serum.

IL-4 expression has been shown to control macrophage inflammatory activities in the pig [40], and IL-4 may positively modulate vaccination mediated clearance of PRRSV [22, 25]. In this study, there were significantly higher levels of IL-4 detected in recombinant virus vaccinated pigs in supernatants of PBMCs at 28 DPI (Fig. 3d), and IL-4 was significantly increased in the sera of pigs vaccinated with recombinant virus at 7 DPC (Fig. 4b). However, the viremia did not show significantly difference compared with parental virus after challenge (Fig. 5b). It is possible that the role of IL-4 may be strain dependent, or it may not have a direct correlation in protecting pigs from HP-PRRSV infection.

IFN- $\gamma$ is a key cytokine that is associated with host cellmediated immunity (CMI) response, which is secreted by natural killer cells and several different $\mathrm{T}$ cell subpopulations [25]. IFN- $\gamma$ reduces PRRSV infection in porcine alveolar macrophages in vitro [47]. A live attenuated PRRSV vaccine that induced high IFN- $\gamma$ secreting cell frequencies protected pigs against viremia $[48,49]$. In this study, piglets immunized with $\mathrm{CH}-1 \mathrm{R}$ strain did not produce higher levels of IFN- $\gamma$, conversely, higher levels of IFN- $\gamma$ were found in piglets infected with HP-PRRSV HuN4 strain (Fig. 4a). Nonetheless, $\mathrm{CH}-1 \mathrm{R}$ strain can offer protection against clinical disease after HP-PRRSV HuN4 infection, and this observation is consistent with our previously published work [50,51]. Therefore, the role of IFN- $\gamma$ in the process of the immune protection to $\mathrm{CH}-1 \mathrm{R}$ vaccination is not clear and need further investigation.

\section{Conclusions}

This is first report to demonstrate the use of PRRSV as a vector to express porcine IL-4. The pIL-4 gene was stably expressed and did not affect the replication capability of the parental virus. The recombinant virus could enhance the T cells immune response by increasing IL-4 expression level and DPT cells ratio in the blood of piglets after HPPRRSV infection. However, it did not significantly improve the efficacy of PRRSV vaccine.

\section{Materials and methods \\ Cells and viruses}

Marc-145 cells (CCTCC, Wuhan, China) were used for rescue and passaging of PRRSV. The cells were propagated in Dulbecco's Modified Eagle's medium (DMEM) with $10 \%$ heat-inactivated FBS (Gibco $\left.{ }^{\circ}, \mathrm{USA}\right)$ at $37^{\circ} \mathrm{C}$ with $5 \% \mathrm{CO}_{2}$. For virus infection and titration, DMEM supplemented with $3 \%$ FBS was used. HP-PRRSV strain HuN4 (GenBank: EF635006) was used in this study for challenge.

\section{Construction of plasmids, transfection and virus passage}

A PRRSV infectious clone, pBAC-CH-1R, was constructed as previously described [52]. The porcine Interleukin-4 (pIL-4) gene (GenBank: HQ236500.1), with a TRS motif and Asis I/ and Mlu I restriction enzyme sites at the 5'and 3 ' - terminal ends, respectively, was constructed using fusion PCR. The PCR fragments were digested and 
inserted into the pBAC-CH-1R vector. The resulting construct, pBAC-CH-1R/pIL-4 was sequenced to confirm the presence and correct cloning of the desired pIL-4 gene sequence (Fig. 1). The primers used for these assays are shown in Table 1.

The pBAC-CH-1R/pIL-4 plasmid was transfected into Marc-145 cells as described previously [52]. Cell culture supernatants were harvested at 4-5 days post transfection and used to infect fresh Marc-145 cells for virus passage.

\section{Growth kinetics and expression of plL-4 of recombinant virus}

Marc-145 cells were infected with the recombinant or the parental virus at a multiplicity of infection (MOI) of 0.01 . $200 \mu \mathrm{L}$ supernatant was collected at $12,24,36,48,72,84$ and $96 \mathrm{~h}$ post infection (hpi) and stored at $-70{ }^{\circ} \mathrm{C}$. Viral titration was determined using the Reed-Muench method. The expression of pIL-4 was determined using a commercial porcine IL-4 ELISA kit (Cloud-Clone Corp, USA).

\section{Indirect immunofluorescence assay (IFA)}

Marc-145 cells grown in 96-well plates were infected with the recombinant or parental virus preparations (0.01 MOI) for $36 \mathrm{~h}$. After fixation using $4 \%$ paraformaldehyde, cells were washed with PBS and incubated with 6D10, a monoclonal antibody to PRRSV $\mathrm{N}$ protein (produced in our lab), or a goat anti-porcine IL-4 polyclonal $\mathrm{Ab}(\mathrm{R} \& \mathrm{D}$ Systems, MN) for $1 \mathrm{~h}$. Cells were then washed with PBS and incubated for $1 \mathrm{~h}$ with a Cy3conjugated goat anti-mouse secondary antibody (Jackson ImmunoResearch Inc, USA) or an Alexa Fluor 488-labeled donkey anti-goat secondary antibody (Abcam, UK), respectively. Images were taken using a Leica confocal microscope.

\section{Western Blotting}

Marc-145 cells were infected with the recombinant or parental viruses $(0.01 \mathrm{MOI})$ for $36 \mathrm{~h}$. The cells were then lysed in RIPA lysis buffer (Beyotime, China). Cell lysates were electrophoresed on $12 \%$ SDS-PAGE gels and transferred onto PVDF membranes (Millipore, USA). The membranes were blocked and incubated for $1 \mathrm{~h}$ with $6 \mathrm{D} 10$ or a goat anti-porcine IL-4 polyclonal Ab (R\&D Systems, MN), then incubated with HRP-conjugated goat anti-mouse IgG (Sigma-Aldrich, USA), or HRP-labeled donkey anti-goat IgG (Sigma-Aldrich), as secondary antibodies, respectively. Immunostained proteins were visualized using ECL Western Blotting Substrate (Beijing Cowin Bioscience, China).

\section{Genetic stability of the recombinant PRRSV}

To investigate whether the inserted pIL-4 gene could be stably maintained in the recombinant virus, the virus was serially passaged 15 times in Marc-145 cells. Viral
RNA was isolated from cells infected with different passaged recombinant viruses (P5, P10 and P15) and RT-PCR was performed using specific primers (Table 1). PCR products were cloned into the pEASY ${ }^{\mathrm{m}}$-Blunt Simple Cloning Vector (TransGen Biotech, China) and the DNA was sequenced.

\section{Animals, vaccination, and HP-PRRSV challenge}

Twenty four-week-old piglets were obtained from a PRRS-free farm in Harbin, China. All piglets were negative for anti-PRRSV antibodies, as assessed by commercial ELISA kit (IDEXX, USA) and PRRSV-free in serum by RT-PCR. Animals were randomly allotted to four groups and housed separately. Piglets were intramuscularly immunized twice with $2 \mathrm{~mL}\left(1 \times 10^{5.5} \mathrm{TCID}_{50} / \mathrm{mL}\right)$ of recombinant virus (group 1) or parental virus (group 2) on 0 and $14 \mathrm{DPI}$.

All piglets were challenged intranasally with $2 \mathrm{~mL} \mathrm{HP}$ PRRSV (HuN4-F5, $1 \times 10^{5.0} \mathrm{TCID}_{50} / \mathrm{mL}$ ) at $28 \mathrm{DPI}$, except those in Group 4, which were given only cell culture medium (uninfected control). Rectal temperatures were documented daily after challenge. Pigs were monitored daily for clinical signs, including dyspnea, lethargy, cough and anorexia. The pigs were euthanized at 28 days postchallenge (DPC). The severity of the clinical signs was evaluated daily after challenge as reported [53]. Briefly, scores ranged from 1 to 4 were determined for behavior, respiration and coughing. Score 1, 2 and 3 represents normal, mild and severe, respectively. Score 4 represents death. To estimate the severity of pathological lesions, gross lesions of each lobe were scored and evaluated as percentage of lung with grossly visible pneumonia [54]. Animal research was conducted under the Animal Welfare and Ethical Guidelines of the Institutional Animal Care Committee.

\section{Circulation antibody titration}

Sera were collected at 14 and 21 DPI and at $0,7,14,21$, and 28 DPC. PRRSV N-specific antibodies were tested using a commercial PRRS ELISA kit (IDEXX, USA) following the manufacturer's instructions.

\section{T lymphocyte proliferation assay}

Peripheral blood mononuclear cells (PBMCs) were isolated from experimental pigs as previously described [55]. Briefly, The PBMCs were diluted to $5 \times 10^{6} / \mathrm{mL}$ in RPMI 1640 supplemented with $10 \%$ FBS and plated in 96-well flat-bottom plates at $100 \mu \mathrm{L}$ per well in triplicate. Subsequently, the purified-PRRS virions $(20 \mu \mathrm{g} / \mathrm{mL})$ as stimulator were added to the cultures, and PHA $(5 \mu \mathrm{g} / \mathrm{mL}$, Sigma-Aldrich, USA) was used as a positive control. After incubation for $72 \mathrm{~h}$ at $37{ }^{\circ} \mathrm{C}$ in $5 \% \mathrm{CO}_{2}, 10 \mu \mathrm{L}$ of WST-8 (Cell Counting Kit-8, Beyotime Biotechnology, China) was added to each well and the cells were incubated for $2 \mathrm{~h}$. 
At the end of the incubation, the absorbance was determined at $450 \mathrm{~nm}$. The stimulation index (SI) was calculated as the ratio of the average optical density (OD) value of wells containing antigen-stimulated cells to the average OD value of wells containing cells cultured with medium alone.

\section{Flow cytometric (FCM) analysis}

Flow cytometric (FCM) analysis was performed to investigate various lymphocyte populations in the blood. Briefly, $50 \mu \mathrm{L}$ of anti-coagulated blood sample was incubated with SPRD-labeled mouse anti-pig CD3e, FITC-labeled mouse anti-pig CD4, and PE-labeled mouse anti-pig CD8 (Southern Biotech, USA), in the dark for 30 min. 1x RBC lysis buffer (BioLegend, San Diego, CA, USA) was added to each sample and gently vortexed immediately for $15 \mathrm{~min}$. Samples were centrifuged at $350 \times \mathrm{g}$ for $5 \mathrm{~min}$ and washed twice with $2 \%$ FBS, before FCM studies were performed using a BD Accuri $^{\text {mm }}$ C6 flow cytometer (BD Biosciences, San Jose, CA, USA). Frequencies of lymphocyte populations were determined from 100,000 events using BD Accuri ${ }^{\mathrm{Tw}}$ C6 software.

\section{Cytokine assays}

Lymphocytes were prepared and stimulated as described above. After incubation for $72 \mathrm{~h}$, culture supernatants from PBMCs of piglets were collected and Th1-type cytokine (IFN- $\gamma$ ) and Th2-type cytokine (IL-4) levels were assessed using commercial ELISA kits (CloudClone Corp, USA).

For the detection of IFN- $\gamma$ and IL-4 production in pigs at various time-points, sera samples were isolated, and cytokines concentrations were determined according to manufacturer's instructions.

\section{Viremia testing}

Total RNA was extracted from sera using Trizol Total RNA Extraction Kit (Shanghai Sangon Biological Engineering Technology \& Services, China), cDNA was synthesized using AMV Reverse Transcriptase XL (TaKaRa, Japan) with oligo (dT) and Quantitative RT-PCR was performed as described previously [50].

\section{Histopathological examination}

All pigs were euthanized at 28 DPC. Lungs were harvested and fixed in $10 \%$ neutral buffered formalin then stained with hematoxylin and eosin (H\&E) for histopathological examination.

\section{Statistical analysis}

GraphPad Prism V.5 software (GraphPad Software, La Jolla, CA, USA) was used for statistical analysis. Results were considered to be statistically significant when the $\mathrm{p}$ value was less than 0.05 , using one-way analysis of variance (ANOVA).

\section{Abbreviations}

PRRSV: Porcine reproductive and respiratory syndrome virus; plL-4: Porcine Interleukin-4; IFA: Indirect immunofluorescent assay; HP-PRRSV: Highly pathogenic PRRSV; DPI: Days post-immunization; DPC: Days post-challenge; FCM: Flow cytometric; TRS6: Transcription regulating sequence for ORF6; Lg: Log10.

\section{Competing interests}

The authors declare that they have no competing interests.

\section{Authors' contributions}

ZJL performed the experiment, arranged the data for statistical analysis and drafted the manuscript. GW, YW, BCH, CZ, QYL, LLL, BYX and PYD participated in animal studies. CBW, XLW, SFS and XHC analyzed the data and revised the manuscript. EMZ designed the study, analyzed the data and revised the manuscript. All authors read and approved the final manuscript.

\section{Acknowledgments}

This study was supported by the National Natural Science Foundation of China $(31430084,31302103)$ and the National High-Tech R \& D Program of China (2011AA10A208).

\section{Author details}

${ }^{1}$ Department of Preventive Veterinary Medicine, College of Veterinary Medicine, Northwest A\&F University, Yangling, Shaanxi 712100, China. ${ }^{2}$ Scientific Observing and Experimental Station of Veterinary Pharmacology and Veterinary Biotechnology, Ministry of Agriculture, Yangling, Shaanxi 712100, China. ${ }^{3}$ State Key Laboratory of Veterinary Biotechnology, Harbin Veterinary Research Institute of Chinese Academy of Agriculture Science, Harbin 150001, China.

Received: 12 May 2015 Accepted: 9 September 2015

Published online: 14 November 2015

\section{References}

1. Pejsak Z, Stadejek T, Markowska-Daniel I. Clinical signs and economic losses caused by porcine reproductive and respiratory syndrome virus in a large breeding farm. Vet Microbiol. 1997;55:317-22.

2. Rossow KD. Porcine reproductive and respiratory syndrome. Vet Pathol. 1998;35:1-20.

3. Wensvoort G, Terpstra C, Pol JM, ter Laak EA, Bloemraad M, de Kluyver EP, et al. Mystery swine disease in The Netherlands: the isolation of Lelystad virus. Vet $Q$. 1991;13:121-30

4. Collins JE, Benfield DA, Christianson WT, Harris L, Hennings JC, Shaw DP, et al. Isolation of swine infertility and respiratory syndrome virus (isolate ATCC VR-2332) in North America and experimental reproduction of the disease in gnotobiotic pigs. J Vet Diagn Invest. 1992;4:117-26.

5. Nelson E, Christopher-Hennings J, Drew T, Wensvoort G, Collins J, Benfield D. Differentiation of US and European isolates of porcine reproductive and respiratory syndrome virus by monoclonal antibodies. J Clin Microbiol. 1993;31:3184-9.

6. Wootton S, Yoo D, Rogan D. Full-length sequence of a Canadian porcine reproductive and respiratory syndrome virus (PRRSV) isolate. Arch Virol. 2000;145:2297-323.

7. Li Y, Wang $X$, Bo K, Wang $X$, Tang B, Yang B, et al. Emergence of a highly pathogenic porcine reproductive and respiratory syndrome virus in the Mid-Eastern region of China. Vet J. 2007;174:577-84.

8. Tian K, Yu X, Zhao T, Feng Y, Cao Z, Wang C, et al. Emergence of fatal PRRSV variants: unparalleled outbreaks of atypical PRRS in China and molecular dissection of the unique hallmark. PLoS One. 2007;2:e526.

9. Tong GZ, Zhou YJ, Hao XF, Tian Z, An TQ, Qiu HJ. Highly pathogenic porcine reproductive and respiratory syndrome, China. Emerg Infect Dis. 2007;13:1434-6.

10. Meulenberg JJ, Hulst MM, de Meijer EJ, Moonen PL, den Besten A, de Kluyver EP, et al. Lelystad virus, the causative agent of porcine epidemic abortion and respiratory syndrome (PEARS), is related to LDV and EAV. Virology. 1993:192:62-72.

11. Meulenberg JJ, Petersen-den Besten A, De Kluyver EP, Moormann RJ, Schaaper WM, Wensvoort G. Characterization of proteins encoded by ORFs 2 to 7 of Lelystad virus. Virology. 1995;206:155-63. 
12. Wu WH, Fang Y, Farwell R, Steffen-Bien M, Rowland RR, Christopher-Hennings J, et al. A 10-kDa structural protein of porcine reproductive and respiratory syndrome virus encoded by ORF2b. Virology. 2001;287:183-91.

13. Johnson CR, Griggs TF, Gnanandarajah J, Murtaugh MP. Novel structural protein in porcine reproductive and respiratory syndrome virus encoded by an alternative ORF5 present in all arteriviruses. J Gen Virol. 2011;92:1107-16.

14. van Aken D, Zevenhoven-Dobbe J, Gorbalenya AE, Snijder EJ. Proteolytic maturation of replicase polyprotein ppla by the nsp4 main proteinase is essential for equine arteritis virus replication and includes internal cleavage of nsp7. J Gen Virol. 2006:87:3473-82.

15. de Vries AAF, Horzinek MC, Rottier PJM, de Groot RJ. The genome organization of the nidovirales: Similarities and differences between arteri-, toro-, and coronaviruses. Semin Virol. 1997;8:33-47.

16. Pasternak AO, Spaan WJM, Snijder EJ. Nidovirus transcription: how to make sense ... ? J Gen Virol. 2006;87:1403-21.

17. Labarque GG, Naumynck HJ, van Woensel PAM, Visser N, Pensaert MB. Efficacy of an American and a European serotype PRRSV vaccine after challenge with American and European wild-type strains of the virus. Vet Res. 2000;31:97.

18. Roca M, Gimeno M, Bruguera S, Segalés J, Díaz I, Galindo-Cardiel IJ, et al. Effects of challenge with a virulent genotype II strain of porcine reproductive and respiratory syndrome virus on piglets vaccinated with an attenuated genotype I strain vaccine. Vet J. 2012;193:92-6.

19. Mateu E, Diaz I. The challenge of PRRS immunology. Vet J. 2008;177:345-51.

20. Park C, Seo HW, Han K, Kang I, Chae C. Evaluation of the efficacy of a new modified live porcine reproductive and respiratory syndrome virus (PRRSV) vaccine (Fostera PRRS) against heterologous PRRSV challenge. Vet Microbiol. 2014; 172:432-42

21. Rehermann $B$. Hepatitis $C$ virus versus innate and adaptive immune responses: a tale of coevolution and coexistence. J Clin Invest. 2009:1 19:1745-54.

22. Li X, Galliher-Beckley A, Huang H, Sun X, Shi J. Peptide nanofiber hydrogel adjuvanted live virus vaccine enhances cross-protective immunity to porcine reproductive and respiratory syndrome virus. Vaccine. 2013;31:4508-15.

23. Peng J, Wang J, Wu J, Du Y, Li J, Guo Z, et al. Positive inductive effect of swine interleukin-4 on immune responses elicited by modified live porcine reproductive and respiratory syndrome virus (PRRSV) vaccine. Viral Immunol. 2013;26:404-14.

24. Tang D, Liu J, Li C, Zhang H, Ma P, Luo X, et al. Positive effects of porcine IL-2 and IL-4 on virus-specific immune responses induced by the porcine reproductive and respiratory syndrome virus (PRRSV) ORF5 DNA vaccine in swine. J Vet Sci. 2014;15:99-109

25. Li X, Galliher-Beckley A, Pappan L, Trible B, Kerrigan M, Beck A, et al. Comparison of host immune responses to homologous and heterologous type II porcine reproductive and respiratory syndrome virus (PRRSV) challenge in vaccinated and unvaccinated pigs. Biomed Res Int. 2014;2014:416727.

26. Kim DY, Calvert JG, Chang KO, Horlen K, Kerrigan M, Rowland RR. Expression and stability of foreign tags inserted into nsp2 of porcine reproductive and respiratory syndrome virus (PRRSV). Virus Res. 2007;128:106-14.

27. Wang L, Zhang K, Lin H, Li W, Wen J, Zhang J, et al. Preparation of North American type II PRRSV infectious clone expressing green fluorescent protein. Biomed Res Int. 2014;2014:368581.

28. Fang Y, Rowland RR, Roof M, Lunney JK, Christopher-Hennings J, Nelson EA. A full-length cDNA infectious clone of North American type 1 porcine reproductive and respiratory syndrome virus: expression of green fluorescent protein in the Nsp2 region. J Virol. 2006;80:11447-55.

29. Xu YZ, Zhou YJ, Zhang SR, Jiang YF, Tong W, Yu H, et al. Stable expression of foreign gene in nonessential region of nonstructural protein 2 (nsp2) of porcine reproductive and respiratory syndrome virus: applications for marker vaccine design. Vet Microbiol. 2012;159:1-10.

30. Han J, Liu G, Wang Y, Faaberg KS. Identification of nonessential regions of the nsp2 replicase protein of porcine reproductive and respiratory syndrome virus strain VR-2332 for replication in cell culture. J Virol. 2007;81:9878-90.

31. Han J, Rutherford MS, Faaberg KS. Proteolytic products of the porcine reproductive and respiratory syndrome virus nsp2 replicase protein. J Virol. 2010;84:10102-12.

32. Han M, Yoo D. Engineering the PRRS virus genome: Updates and perspectives. Vet Microbiol. 2014;174:279-95.

33. Pei Y, Hodgins DC, Wu J, Welch S-KW, Calvert JG, Li G, et al. Porcine reproductive and respiratory syndrome virus as a vector: immunogenicity of green fluorescent protein and porcine circovirus type 2 capsid expressed from dedicated subgenomic RNAs. Virology. 2009;389:91-9.
34. Wang L, Hou J, Gao L, Guo XK, Yu Z, Zhu Y, et al. Attenuation of highly pathogenic porcine reproductive and respiratory syndrome virus by inserting an additional transcription unit. Vaccine. 2014;32:5740-8.

35. Lawson SR, Li Y, Patton JB, Langenhorst RJ, Sun Z, Jiang Z, et al. Interleukin-1 $\beta$ expression by a recombinant porcine reproductive and respiratory syndrome virus. Virus Res. 2012;163:461-8.

36. Sang Y, Shi J, Sang W, Rowland RR, Blecha F. Replication-competent recombinant porcine reproductive and respiratory syndrome (PRRS) viruses expressing indicator proteins and antiviral cytokines. Viruses. 2012;4:102-16.

37. Yu L, Zhou Y, Jiang Y, Tong W, Yang S, Gao F, et al. Construction and in vitro evaluation of a recombinant live attenuated PRRSV expressing GM-CSF. Virol J. 2014;11:201.

38. Kimman TG, Cornelissen LA, Moormann RJ, Rebel JM, Stockhofe-Zurwieden N Challenges for porcine reproductive and respiratory syndrome virus (PRRSV) vaccinology. Vaccine. 2009;27:3704-18.

39. Rompato G, Ling E, Chen Z, Van Kruiningen H, Garmendia AE. Positive inductive effect of IL-2 on virus-specific cellular responses elicited by a PRRSV-ORF7 DNA vaccine in swine. Vet Immunol Immunopathol. 2006;109:151-60.

40. Murtaugh MP, Johnson CR, Xiao Z, Scamurra RW, Zhou Y. Species specialization in cytokine biology: is interleukin-4 central to the $T(H) 1-T(H) 2$ paradigm in swine? Dev Comp Immunol. 2009;33:344-52.

41. Charerntantanakul W. Adjuvants for porcine reproductive and respiratory syndrome virus vaccines. Vet Immunol Immunopathol. 2009;129:1-13.

42. Shimizu M, Yamada S, Kawashima K, Ohashi S, Shimizu S, Ogawa T. Changes of lymphocyte subpopulations in pigs infected with porcine reproductive and respiratory syndrome (PRRS) virus. Vet Immunol Immunopathol. 1996;50:19-27.

43. Dwivedi V, Manickam C, Binjawadagi B, Linhares D, Murtaugh MP, Renukaradhya GJ. Evaluation of immune responses to porcine reproductive and respiratory syndrome virus in pigs during early stage of infection under farm conditions. Virol J. 2012:9:45.

44. Carvalho LH, Sano G, Hafalla JC, Morrot A, Curotto de Lafaille MA, Zavala F. IL-4-secreting CD4+ T cells are crucial to the development of CD8+ T-cell responses against malaria liver stages. Nat Med. 2002;8:166-70.

45. Paliard X, Malefijt RW, de Vries JE, Spits H. Interleukin-4 mediates CD8 induction on human CD4+ T-cell clones. Nature. 1988;335:642-4.

46. Brod SA, Purvee M, Benjamin D, Hafler DA. Frequency analysis of CD4 + CD8+ T cells cloned with IL-4. Cell Immunol. 1990;125:426-36.

47. Gaudreault N, Rowland RR, Wyatt CR. Factors affecting the permissiveness of porcine alveolar macrophages for porcine reproductive and respiratory syndrome virus. Arch Virol. 2009;154:133-6.

48. Diaz I, Darwich L, Pappaterra G, Pujols J, Mateu E. Different European-type vaccines against porcine reproductive and respiratory syndrome virus have different immunological properties and confer different protection to pigs. Virology. 2006;351:249-59.

49. Park C, Seo HW, Han K, Kang I, Chae C. Efficacy of a new modified live porcine reproductive and respiratory syndrome virus (PRRSV) vaccine (Fostera PRRS) against heterologous PRRSV challenge. Vet Microbiol. 2014;172(3-4):432-42.

50. Wang G, Song T, Yu Y, Liu Y, Shi W, Wang S, et al. Immune responses in piglets infected with highly pathogenic porcine reproductive and respiratory syndrome virus. Vet Immunol Immunopathol. 2011;142:170-8.

51. Yu Y, Cai X, Wang G, Kong N, Liu Y, Xiao Y, et al. Anti-idiotypic antibodies reduce efficacy of the attenuated vaccine against highly pathogenic PRRSV challenge. BMC Vet Res. 2014;10:39.

52. Wang C, Huang B, Kong N, Li Q, Ma Y, Li Z, et al. A novel porcine reproductive and respiratory syndrome virus vector system that stably expresses enhanced green fluorescent protein as a separate transcription unit. Vet Res. 2013;44:104.

53. Wang $Y$, Liang $Y$, Han J, Burkhart KM, Vaughn EM, Roof MB, et al Attenuation of porcine reproductive and respiratory syndrome virus strain MN184 using chimeric construction with vaccine sequence. Virology. 2008;371:418-29.

54. Halbur PG, Miller LD, Paul PS, Meng XJ, Huffman EL, Andrews JJ. Immunohistochemical identification of porcine reproductive and respiratory syndrome virus (PRRSV) antigen in the heart and lymphoid system of three-week-old colostrum-deprived pigs. Vet Pathol. 1995;32:200-4.

55. Wongyanin P, Buranapraditkun S, Chokeshai-Usaha K, Thanawonguwech R, Suradhat S. Induction of inducible CD4 + CD25 + Foxp3+ regulatory T lymphocytes by porcine reproductive and respiratory syndrome virus (PRRSV). Vet Immunol Immunopathol. 2010;133:170-82. 